\title{
Concepciones de docentes sobre evaluación del aprendizaje en básica primaria
}

\section{Conceptions of teachers on learning assessment on basic primary}

\author{
Dina Margarita Bravo Colón \\ Licenciada en Educación Básica Primaria con Énfasis en Ciencias Sociales \\ Centro Educativo El Yeso. Morroa-Sucre. Colombia. \\ bdinamargarita@yahoo.es
}

Hannia Karina Hernández Salcedo Licenciada en Lengua Castellana y Comunicaciones. Institución Educativa Indígena Sabanas de la Negra. Sampues-Sucre. Colombia. hkhernandez.148@hotmail.com

\section{Resumen}

Esta investigación cualitativa con diseño de investigación acción tuvo como propósito analizar las concepciones docentes sobre evaluación del aprendizaje para mejorar el desempeño de los estudiantes de educación básica en el ciclo primaria. Los docentes con la conformación de una comunidad de reflexión acción (CRA) hicieron revisión de las practicas evaluativas y de teorías que reencaminaron la resignificación del proceso evaluativo a nivel de aula e institucional, a través de estas reflexiones se alcanzó el reconocimiento de la importancia del actuar docente en el desarrollo de un proceso de enseñanza - aprendizaje formativo.

Palabras clave: aprendizaje, enseñanza, evaluación, competencias, concepciones de evaluación.

\begin{abstract}
This qualitative research with action research design had the purpose of analyzing the conceptions of teachers on learning assessment to improve the performance of the students of basic education in the primary cycle. Teachers, with the constitution of a community of action research reflection (CRA) made a review of the assessment practices and theories that rerouted the re-signification of the assessment process at a classroom and institutional level, reaching the recognition of the importance of the actions of the teacher in the development of a teaching process was reached through these reflections - formative learning.
\end{abstract}

Key Words: learning, teaching, assessment, competences, assessment conceptions.

\section{Introducción}

\section{Concepciones docentes}

En lo concerniente al accionar docente en el aula existen múltiples vías de investigación, lo que a consecuencia resulta en una diversidad de conceptos y términos para referirse a realidades similares que emplean diferentes metodologías y enfoques. Sin embargo en este estudio al revisar la literatura relacionada con el tema y en relación con el propósito de investigación, se optó por la expresión concepciones como la palabra que engloba lo relacionado a lo que piensa, siente y hace el docente con respecto a la evaluación del aprendizaje y de qué manera se puede potencializar el proceso formativo de los estudiantes mediante ese hacer evaluativo es decir mediante el actuar docentes. Como señala Fullan (citado por Mellado, 2011) "los cambios en educación dependen de lo que piensan y hacen los profesores, algo tan simple y a la vez tan complejo" (p.11).

Para el análisis de las concepciones docentes, se incluyeron aportes desde tres perspectivas: pensamiento del profesor, creencias y teorías implícitas. Mediante el primer enfoque, se busca estudiar el pensamiento del profesor, su estructura y la relación que existe entre lo que piensa y hace, Así mismo, la correspondencia entre contexto-procesos-acciones y como se vinculan al pensamiento evidenciando sus teorías y creencias; se concibe al docente como investigador que indaga, reflexiona en y sobre su actuar; al respecto Pérez \& Gimeno (1988) hace una clasificación de investigaciones tocantes al tema, en dos corrientes: en enfoque cognitivo en el cual autores como Jackson, Clark y Yinger (citados por O'shanahan, 1996) hacen la distinción entre las fases pre activa, 
interactiva y pos activa de la enseñanza, división que fue utilizada por autores como Crist, Marx y Peterson 1974 (citados por Antón, 2012 ) quienes evidenciaron que el pensamiento de los docentes antes y después de la interacción en la clase es diferente al empleado durante su accionar en el aula. Las fases señalan distintos momentos con sus actividades propias: En la fase pre-activa se planifica y organiza la enseñanza reflejando el pensamiento organizado y sistemático del docente al momento de planear y organizar su clase. En la fase interactiva, el docente interactúa entre los estudiantes y el conocimiento, buscando desarrollar habilidades y actitudes mediante la propuesta de enseñanza. Y en la fase pos activa el docente realiza un análisis crítico de su trabajo realizado en el aula y del desempeño de los estudiantes, teniendo en cuenta lo planeado, es decir evalúa su trabajo, con el fin de mejorar las prácticas y preparar nuevas experiencias. De esta manera, la reflexión es útil para analizar y mejorar conscientemente sus creencias e intereses y tomar decisiones. Dada las tres fases, se deduce del actuar docente un estado cíclico en el proceso de enseñanza aprendizaje evaluación. Este enfoque cognitivo según Pérez \& Gimeno (1988), ha centrado su análisis en las atribuciones del profesor sobre las causas del desempeño de los alumnos, es decir sobre el éxito o fracaso de los discentes y en menor medida en las teorías implícitas del profesor.

Por otra parte el enfoque alternativo basado conceptualmente en el paradigma de las ciencias sociales, concibe al hombre como agente de sus propias actuaciones, por ello para comprender las acciones del docente y sus repercusiones, se hace necesario indagar sobre las interpretaciones de estas, tanto a agentes como receptores. (Marrero, 2010), (Perrenoud, 2007), (Mellado, 1996).

Desde la segunda perspectiva, atendiendo a las características propuestas por Pajares (citado por Marrero, 2010) sobre las creencias de los profesores: naturaleza, organización y flexibilidad, se busca comprender el actuar docente contrastando lo que piensa, hace y dice (Moreno \& Azcárate, 2003); Y en la tercera o última perspectiva estudiada se permite hacer explícito aquello que no es accesible a la conciencia, que aunque su origen se da mediante la interacción social y cultural, se construye de forma personal; influyendo en las acciones que realiza (conocimiento, enseñanza, aprendizaje, evaluación, entre otras), pues son activadas dependiendo la tarea a realizar (Marrero, 2010), (Antón, 2012); en relación Díaz (2005) se refiere a las teorías implícitas desde la didáctica como "representaciones mentales que hacen parte del sistema de conocimiento de un individuo las cuales intervienen en la comprensión, memoria, razonamiento y planificación de la acción" (p.45). De esta manera, se constituyen en un fenómeno ineludible en la realidad de la enseñanza, las cuales están inmersas en su diseño, la construcción de las tareas académicas, en la toma de decisiones y también en la evaluación, que ordena, regula e interviene en los procesos de enseñanza y del aprendizaje; específicamente en el caso de los profesores, se consideran como experiencias prácticas construidas en el escenario de la cultura escolar, permitiéndole percibir, interpretar, decidir, actuar y valorar (Pérez \& Gimeno, 1988).

Por otra parte, el termino concepciones en esta investigación está en consonancia con la síntesis elaborada por Moreno y Azcarate (2003) "las concepciones son organizadores implícitos de los conceptos, de naturaleza esencialmente cognitiva y que incluyen creencias, significados conceptos proposiciones, reglas, imágenes mentales, preferencias, etc., que influyen en lo que se perciben y en los procesos de razonamiento que se realizan. El carácter subjetivo es menor en cuanto se apoyan sobre un sustrato filosófico" (p.267) Conocer las concepciones no solo ayudaran a comprender mejora algunas de las dificultades del sistema educativo colombiano y del centro en el cual se realiza este estudio para responder a las demandas de las llamadas sociedad del conocimiento sino en la medida en que ese conocimiento contribuya a comprender mejor esos cambios puede ayudar a promoverlos o dirigirlos en la dirección deseada (Pozo, Scheuer, Pérez, Mar, \& Martín, 2006). En referencia, Vergara (2011) afirma "la forma de evaluar devela el concepto que el docente tiene de lo que es enseñar y aprender" (p.4) manifestando aspectos fundamentales del docente como los conocimientos profesionales y sus creencias, además, según este autor causan efecto directo en el aprendizaje de los estudiantes.

\section{Evaluación del aprendizaje}

Ante la existencia de variedad de aportaciones por muchos autores demostrando riqueza conceptual y manejo que han hecho de este constructo en las últimas décadas, en este estudio de acuerdo a su contexto se pretende dar respuesta a lo que se considera evaluación, para que evaluar, cómo y cuándo evaluar; por lo cual se toman aportes de Scriven (citado por Delgado, 2010), (Córdoba, 2006), (Monereo, 1999) y se asume la evaluación como: el conjunto de acciones acordes a un contexto y tiempos particulares que permiten al estudiante y docente autorregular, comprender y evidenciar su desempeño en el proceso formativo. Puesto que busca enfocarse en los sucesos que día a día transcurren en el aula, en observar y buscar información para llegar a reflexionar y comprender cómo se está aprendiendo; qué se necesita aprender; dónde se necesita fortalecer conceptos y procesos, enriqueciendo el proceso formativo. Dado lo anterior y las características del contexto donde se desarrolla este estudio se considera pertinente un tipo de evaluación que sea Formadora, motivadora, orientadora, contextualizada, participativa y continua las cuales en conjunto vinculadas con la evaluación formativa.

En la educación primaria actualmente la evaluación se regula mediante decreto 1290 de 2009 , enfocada en propósitos formativos, convirtiéndose de manera simultánea en beneficio para quien enseña y quien 
aprende, al respecto Álvarez (2007) se refiere al maestro y el estudiante como el sujeto que aprende con ella y a través de ella, estableciendo un equilibrio entre la función pedagógica y social. De esta manera, el concepto sobre que es evaluar debe ser coherente con los métodos, las técnicas, instrumentos y estrategias de evaluación, que minuciosamente el docente seleccione para su puesta en acción, contribuyendo a mejorar el proceso evaluado. Ahora bien, teniendo en cuenta que los propósitos en la evaluación del aprendizaje son variados dependiendo el tipo de evaluación, Casanova (1998) establece unos criterios para su mejor uso con relación al propósito o finalidad (sumativa y formativa), según el momento (inicial, procesual y final) y el agente evaluador (autoevaluación, coevaluación y heteroevaluación) (p.81) Al respecto Brown \& Glasner (2007) afirman "la única cosa útil que nosotros como profesores podemos hacer para influenciar positivamente los procesos de aprendizaje y enseñanza, es elegir correctamente el diseño de la estrategia de evaluación" (p.24) lo cual implica calidad de estrategias evaluativas a emplear en la práctica de aula, como también proveer de buena información de las ejecuciones realizadas por los estudiantes y estar orientada a que el estudiante construya la respuesta y de razón del porqué de la misma, más que a reproducirla o seleccionarla.

Por otra parte, se consideran métodos evaluativos que difieren de los tradicionales: Exámenes a libro abierto, que demuestra lo que los estudiantes pueden hacer con la información. Estudio de caso, en el cual se prueba la síntesis y el análisis. Simulaciones, permitiendo demostrar sus capacidades y habilidades aplicadas en entornos reales. Exámenes de preguntas de múltiple elección, en los cuales pueden demostrar comprensión en diversos temas. Según Brown \& Glasner et al. (2007) refiere que una manera acertada de elegir un método de evaluación es pensar en lo que realmente los estudiantes quieren demostrar con lo que saben. De igual forma al elegir un instrumento se debe tener en cuenta que no es solo reemplazar lo que se viene empleando sino la filosofía subyacente Harris and Bell, (citado por Brown \& Glasner, 2007). Por lo cual se hace necesario antes de una elección o diseño generar reflexión alrededor de ciertos interrogantes como: el ¿Por qué se está evaluando?, ¿qué y cómo se está evaluando?, ¿cuándo se debería evaluar? Al respecto Álvarez (2007) afirma más que un instrumento para evaluar, lo importante es el tipo de conocimiento que pone a prueba, el tipo de preguntas que se formulan, el tipo de cualidades (mentales o prácticas) que se exigen y las respuestas que se espera obtener según el contenido de las preguntas o problemas que se formulan (p.70), esto implica el propósito con el cual se evalúa.

\section{Problema}

Alrededor del proceso evaluativo se vienen acrecentando ciertas disparidades entre el qué y cómo se enseña y el cómo se evalúa, en lo cual hay mayor interés y atención en los resultados que se obtienen y no en el proceso que se realiza, el cual si es utilizado desde una perspectiva formativa valoraría no solo el conocimiento sino la aplicabilidad del mismo. Hay diferentes elementos que su análisis pertinente ayudaría en ello como son los resultados de pruebas externas e internas, situación del sistema evaluativo y curricular de la institución, la evaluación y promoción académica, experiencias pedagógicas de aula y expresiones docentes.

La información suministrada en exámenes externos está indicando que existe una problemática generalizada la cual afecta la calidad educativa, pero también contribuye en la toma de decisiones sobre la necesidad de realizar acciones encaminadas a mejorar los procesos de enseñanza - aprendizaje - evaluación desde el contexto escolar, desarrollando competencias que se conviertan en las bases auto formativas de los estudiantes para que realmente puedan seguir aprendiendo a lo largo de la vida; de esta manera en el contexto educativo se estaría cumpliendo con la finalidad de "brindar información para que los maestros vuelvan a mirar sus procesos de enseñanza y los educandos enfaticen y consoliden sus procesos de aprendizaje" (Castro, Martínez, \& Figueroa, 2009, p.17).

En Colombia el Ministerio de Educación Nacional (MEN) ha diseñado directrices (decreto 230 de 2002 y decreto 1290 de 2009 ) donde se ha promovido la valoración integral de los desempeños de los estudiantes, siendo evidente que los docentes han estado concertando con los conceptos de evaluación y competencias hace más de doce años por lo que se hace pertinente que en el análisis de las concepciones de docentes sobre evaluación del aprendizaje se contraste la interpretación que hacen del decreto con sus prácticas evaluativas

El Centro Educativo el Yeso como sujeto de estudio de esta investigación no es ajeno a esta problemática, al revisar documentos institucionales se identificaron dificultades en el sistema evaluativo y curricular como: La autoevaluación institucional de 2011 y 2012 arroja resultados incoherentes con la realidad en el aula, con respecto a la aplicación de la norma vigente en el proceso de evaluación del aprendizaje. El Sistema Institucional de Evaluación no se aplica según la norma vigente: docentes asumen criterios individuales para evaluar el aprendizaje, estudiantes y padres de familia desconocen sus responsabilidades, derechos y deberes con respecto al desempeño de estudiantes. La estadística de los años 2011 al 2012 refleja altos índices de reprobación y deserción: de los 80 estudiantes matriculados en 2011 un 16\% reprobaron el año escolar y un 15\% desertaron. En 2012 un 19\% de 74 estudiantes matriculados reprobaron su grado y un 7\% desertaron. El Proyecto Educativo Institucional describe el enfoque pedagógico histórico social en el que se encuentra fundamentado el modelo socio constructivista que orienta el proceso enseñanza - aprendizaje, si se llevara a la práctica permitiría la formación y valoración del saber, saber hacer, ser y convivir. La planeación curricular está basada en procesos cognitivos por competencias, indicadores de desempeño, 
centrados en el ser, saber y el hacer, especificado por asignaturas y con base en los Estándares para la excelencia educativa, pero en las planeaciones de clases solo se evidencian actividades relacionadas al saber conocer. El proceso de evaluación y promoción académica se aplica de forma incoherente a lo estipulado en los artículos 3, 4, 5 y 12 del decreto 1290 del 2009 que corresponde al qué y cómo evaluar, dado que la meta principal para los docentes al momento de evaluar es contraria a la valoración de habilidades, capacidades y destrezas en sus estudiantes, centrándose en el concepto y su aplicación; direccionan el cómo hacerlo solo a las formas de evaluación mas no al sentido de integralidad de esos saberes al ser aplicados en diversos contextos. De esta manera si se comprendiera el sentido de continuidad en la evaluación como lo establece el Ministerio de Educación Nacional (MEN) la evaluación llegaría a ser un elemento de aprendizaje para todos; pues al momento de enseñar, se evalúa y se aprende (Castro, Martínez, \& Figueroa, 2009).

Expresiones de docentes, estudiantes y padres de familia dan testimonio de creencias frente a la evaluación, los docentes manifiestan: "Los estudiantes tienen dificultades en el aprendizaje en procesos de análisis, reflexión, argumentación, interpretación, resolución de problemas y proponer soluciones", "El bajo desem-peño en el aprendizaje de los estudiantes se debe al efimero apoyo de los padres de familia hacia el proceso formativo de sus hijos". Los estudiantes afirman sentir temor al momento de ser evaluados por la calificación que pueda colocar su profesor, La nota o calificación que asigna el maestro a una evaluación determina cuanto ha aprendido en el transcurso del mes, periodo $o$ año para luego ser promovido a otro grado, promocionado a otro nivel educativo o reprobado. Los padres de familia: "El maestro que enseña es aquel que logra que sus alumnos repitan lo que el explico", "las preguntas en un examen deben ser de lo que está en el cuaderno", "el examen escrito es la única forma de evaluar que tanto sabe mi hijo", "El maestro es quien sabe que enseñar y como evaluar".

Esta situación presente en el proceso evaluativo no es nueva, porque varias investigaciones realizadas alrededor del mundo señalan esa desarticulación que existe en la forma de evaluar entre lo que se piensa, se dice y se hace, (Moreno \& Ortiz, 2008), (Vidales, 2005) (Mellado, 1996). De hecho en el contexto de la investigación se ha intentado analizar por parte de docentes esta realidad, pero no ha sido posible pues se hace mayor énfasis en las dificultades de los estudiantes, originadas por su actitud o situaciones externas al ámbito escolar.

\section{Planteamiento del problema}

Lo anterior lleva a plantear el siguiente interrogante: ¿Cómo se relacionan las concepciones docentes sobre evaluación del aprendizaje con el desempeño de estudiantes del Centro Educativo el Yeso en el municipio de Morroa Sucre?

\section{Justificación}

La calidad de la educación en Colombia ha sido uno de los mayores retos por mejorar de gobiernos y del sistema educativo, atendiendo la visión que se tenga de calidad y los fines que persigue el proceso formativo; el Ministerio de Educación Nacional (MEN) en su intento ha empleado la evaluación como herramienta de apoyo y conocimiento para verificar si se está trabajando en la dirección adecuada conforme a lo establecido en la normatividad vigente (Constitución política de Colombia en su artículo 67, de conformidad con la Ley General de educación (Ley 115) en su artículos 79 y 80 , decreto 1290 de 2009 ).

Sin embargo, es reiterativo el no funcionamiento de estas directrices frente a la evaluación del aprendizaje en establecimientos educativos como el Centro Educativo el Yeso y sus sedes asociadas, por lo cual se hace necesario intervenir en el proceso evaluativo. Es a través de la evaluación donde el docente puede reflexionar sobre sus prácticas de aulas, no solo en la forma como aprenden los estudiantes sino en la calidad de lo que aprenden, atendiendo las acciones formativas de enseñanza y evaluación de manera continua mediante la autocritica y autocorrección que apunte a una mejor organización, planeación y valoración del proceso enseñanza-aprendizaje, enriqueciendo el quehacer pedagógico del docente y retroalimentando sus concepciones para su cualificación. Así mismo, desde el aula la evaluación propicia ayuda al alumnado para que aprenda de y con ella, haciendo del proceso evaluativo una acción de conocimiento, de orientación oportuna evitando que cualquier error detectado sea definitivo y se convierta en fracaso escolar. Además, en la medida en que se haga buen uso de la evaluación del aprendizaje y se comprenda su finalidad, el padre de familia se mantendrá informado de manera permanente sobre el proceso de formación en el aula y podrá participar en la toma de decisiones en consenso con los demás actores de la comunidad educativa.

Por lo cual es un tema de investigación que contribuye a una necesidad relevante, meta principal y reto trazado por las políticas educativas colombianas a nivel regional, nacional e internacional en los últimos años como es la calidad de la educación; se considera pertinente frente a la problemática actual que presentan los estudiantes en el bajo desempeño de competencias en los diferentes niveles educativos; además, guarda coherencia con los requerimientos del decreto 1290 del 2009 el cual busca promover el uso complementario de las evaluaciones externas para que al igual que las internas apunten al desarrollo de competencias en el estudiantes acorde a los referentes de calidad EBC (Estándares Básicos de Competencias); de igual forma demanda el sentido formativo de la evaluación en el aula, mediante mejoramiento continuo en la formación del estudiantes pero también en el docente permitiendo reflexionar y tomar conciencia de la forma de entender y de practicar la evaluación en el aula de clases, llevando a analizar los fundamentos teóricos 
que subyacen en el pensamiento de quienes la orien$\tan$.

\section{Metodología}

Esta investigación cualitativa, se interesa por la vida de las personas, por sus sentimientos, comportamientos, experiencias, por sus interacciones, por sus acciones, los interpreta de manera contextual ubicándolo en el espacio que tiene lugar. Tratando de comprenderlos y explicarlos acudiendo a la causa local convirtiéndose está en un rasgo epistemológico de esta perspectiva. La modalidad de investigación empleada desde una perspectiva explicativa es la investigación acción. Esta según su forma de producir conocimiento se sitúa en el paradigma critico-social cuyo interés es indagar, comprender y transformar. Convirtiéndose en una alternativa metodológica innovadora y de transformación social (Vasilachis, 2006), (Sandoval, 1996); (Valles, 1997). Se caracteriza por la participación activa y democrática en la toma de decisiones para generar conocimiento mediante la investigación, logrando renovar la realidad mediante la acción (Elliot, 2000), (Lafourcade, 1992), (Elliott y Kemmis; citado por Murillo, 2011).

El diseño metodológico implementado en este estudio, se caracteriza por su carácter cíclico que implica un espiral dialéctica entre la acción y la reflexión integrándose y complementándose, siendo un proceso flexible e interactivo en todas las fases. Las categorías a analizar son concepciones de evaluación docente en la que se tiene en cuenta criterios como: Teoría Evaluativa, metodología, didáctica, agente evaluador y motivación; en relación con el desempeño de estudiantes cuyos criterios son: motivación, participación en clase, desarrollo de actividades y relación maestroestudiante.

Según el modelo utilizado se emplean 3 fases y en cada uno de ellas se realizan unos momentos: observaciónacción, reflexión-acción y valoración-acción. Los submomentos ayudan a organizar el proceso, transformándose en nuevos de ellos. La palabra acción se repite en cada uno de ellos, dado que según Murillo (2011) en la investigación acción el énfasis se pone en la acción más que en la investigación (p.21), pues la investigación se constituye en una forma de acción y en este caso, cada submomentos constituye en sí una acción que se conecta con otro.

\section{Población}

La población estudiada la constituyen 9 docentes: 4 laboran en el Centro Educativo El Yeso y 5 en las seis sedes asociadas, todos atienden aulas con modalidad multigrados en el nivel de educación básica ciclo de primaria. Ubicada en el Corregimiento el Yeso, perteneciente al municipio de Morroa en el departamento de Sucre.

\section{Muestra}

Para esta investigación el grupo de docentes involucrado quedó constituido por 6 docentes, a quienes se les asigno un código (I, D1, D2, D3, D4, D5), los cuales voluntariamente participaron de la investigación una vez se informó sobre el propósito de la misma y los beneficios de analizar lo que piensan, sienten, hacen y dicen sobre evaluación del aprendizaje, conformando un equipo de trabajo al que llamaron Comunidad de Reflexión-Acción CRA.

\section{Proceso metodológico}

Tabla 1. Fases y submomentos de la Investigación Acción

\begin{tabular}{|c|c|c|c|c|}
\hline \multirow[b]{3}{*}{$\begin{array}{l}\text { FASE } \\
\text { DE } \\
\text { EXPLOR } \\
\text { ACION }\end{array}$} & \multirow[t]{2}{*}{ OBJETIVO } & \multicolumn{3}{|l|}{ SUBMOMENTOS } \\
\hline & & $\begin{array}{l}\text { OBSERVACION- } \\
\text { ACEION }\end{array}$ & $\begin{array}{l}\text { REFLEXION- } \\
\text { ACCION }\end{array}$ & $\begin{array}{l}\text { VALORACION- } \\
\text { ACOION }\end{array}$ \\
\hline & $\begin{array}{l}\text { Lograr la } \\
\text { participación activa } \\
\text { y responsable de } \\
\text { todos los docentes } \\
\text { en la observación } \\
\text { y la descripción de } \\
\text { prácticas de aula } \\
\text { que permitan la } \\
\text { identificación de } \\
\text { sus concepciones } \\
\text { de docentes sobre } \\
\text { evaluación del } \\
\text { aprendizaie. }\end{array}$ & $\begin{array}{l}\text { Areas por mejorar } \\
\text { Revisión de } \\
\text { documentos } \\
\text { institucionales } \\
\text { Descripción de } \\
\text { clases } \\
\text { Observación de } \\
\text { clase } \\
\text { Aplicación de } \\
\text { entrevista a } \\
\text { docentes }\end{array}$ & $\begin{array}{l}\text { Contraste } \\
\text { interpretación de } \\
\text { la información } \\
\text { suministrada por } \\
\text { instrumentos } \\
\text { aplicados }\end{array}$ & $\begin{array}{l}\text { Elaboración de } \\
\text { informe } \\
\text { correspondiente al } \\
\text { contraste realizado } \\
\text { entre instrumentos } \\
\text { aplicados. }\end{array}$ \\
\hline $\begin{array}{l}\text { FASE } \\
\text { DE } \\
\text { EJECUC } \\
\text { ION }\end{array}$ & $\begin{array}{l}\text { Aprender a } \\
\text { reconocer las } \\
\text { inconsistencias } \\
\text { existentes en las } \\
\text { prácticas de aula a } \\
\text { traves de la } \\
\text { revisión teórica en } \\
\text { contraste con la } \\
\text { revisión de la } \\
\text { documentación } \\
\text { institucional. }\end{array}$ & $\begin{array}{l}\text { Socialización y } \\
\text { desarrollo de guías } \\
\text { (modelo } \\
\text { pedagógico, } \\
\text { modelos } \\
\text { evaluativos, decreto } \\
\text { 1z9o, la instrucción } \\
\text { en las métodos e } \\
\text { instrumentos } \\
\text { evaluativos, } \\
\text { evaluacion de } \\
\text { competencias) }\end{array}$ & $\begin{array}{l}\text { Análisis detallado } \\
\text { del SlE basado } \\
\text { en una } \\
\text { evaluación } \\
\text { formativa } \\
\text { integral en } \\
\text { relación con las } \\
\text { prácticas } \\
\text { evaluativas del } \\
\text { docente. }\end{array}$ & $\begin{array}{l}\text { Informe de } \\
\text { fortalezas y } \\
\text { aspectos por } \\
\text { mejorar del SIE } \\
\text { teniendo en cuenta } \\
\text { los criterios teóricos } \\
\text { revisados en } \\
\text { relación con las } \\
\text { práticas } \\
\text { evaluativas del } \\
\text { docente. }\end{array}$ \\
\hline $\begin{array}{l}\text { FASE } \\
\text { DE } \\
\text { VALORA } \\
\text { OIONY } \\
\text { SISTEM } \\
\text { ATIZACI } \\
\text { ON }\end{array}$ & $\begin{array}{l}\text { Valorar el cambio } \\
\text { generados en las } \\
\text { concepciones } \\
\text { evaluatias de los } \\
\text { docentes en } \\
\text { relacion con el } \\
\text { desempeño en los } \\
\text { estudiantes } \\
\text { refleiado en sus } \\
\text { prácticas de aula. }\end{array}$ & $\begin{array}{l}\text { Observaciones de } \\
\text { prácticas evaluativas } \\
\text { teniendo en cuenta } \\
\text { los criterios } \\
\text { sugeridos en el SIE }\end{array}$ & $\begin{array}{l}\text { Contraste entre } \\
\text { observaciones de } \\
\text { prácticas } \\
\text { evaluativas } \\
\text { iniciales y en } \\
\text { desarrollo. }\end{array}$ & $\begin{array}{l}\text { Valoración del } \\
\text { cambio en las } \\
\text { concepciones } \\
\text { evaluativas de los } \\
\text { docentes en } \\
\text { relacion con el } \\
\text { desempeño } \\
\text { generado los } \\
\text { estudiantes... }\end{array}$ \\
\hline
\end{tabular}




\section{Resultados}

Aquí se hace anotación de los principales resultados en cada fase y submomentos que la conforman.

\section{Informe primera fase: exploración}

El alcance de esta fase fue el reconocimiento del tipo de concepciones predominante en las prácticas evaluativas de los docentes lo cual se demuestra en el desarrollo de cada uno de los submomentos en que se subdivide esta fase:

Observación-acción: Las acciones realizadas por la CRA en este submomentos centran la atención en la motivación reflejada en expresiones, determinación de necesidades en torno al proceso evaluativo, revisión documental (descripción de clases y documentos institucionales), observación de clases y en entrevista. Se analizo primeramente la información sobre expresiones de docentes como: "Cuente seño que nosotros apoyaremos este proceso de investigación, porque me hace falta aprender mucho sobre evaluación"(D1), "Me interesa conocer más sobre las últimas tendencias en evaluación y sobre las competencias, creo que en esto presentamos falencias"(D5) entre otras. Lo cual permitió notar que hay conciencia en los docentes de una necesidad institucional en torno a la evaluación que se está llevando en los procesos curriculares. Además orientó la investigación hacia la claridad con respecto a las repercusiones que tales dificultades generaron en el desempeño de los estudiantes en toda acción pedagógica- evaluativa realizada, así esta acción de expresar lo que sienten frente al proceso en palabras de Pérez \& Gimeno, (1988) "deja impresa las huellas de su pensamiento en cada una de ellas" (p.17). Así mismo, con respecto a las necesidades que se debían mejorar frente al proceso de evaluación del aprendizaje y de acuerdo a cada criterio de análisis se deduce con respecto a las concepciones de evaluación docente que los maestros justifican su actuar por las debilidades en el sistema de evaluación institucional, la ausencia de la construcción de un plan de evaluación compartidos por todos en la básica primaria, unificación de criterios de evaluación y el desconocimiento del modelo pedagógico del establecimiento educativo, así el interés se ve centrado en el contexto donde condicionantes institucionales y sociales influyen en el establecimiento de las practicas del docente Erickson \& Hammersley (citado por O'shanahan, 1996) lo cual requiere de un actuar reflexivo que guía un proceso de decisión basado en la responsabilidad y autonomía de quien enseña (Perrenoud, 2007). En la revisión del PMI, se revela que aunque se hace una evaluación en el plan de mejoramiento no se evidencia un proceso que enriquezca la adecuada evaluación formativa institucional que permita el desarrollo y seguimiento de las dimensiones del ser humano, pues se evidencia que no se ciñen a criterios establecidos. También, se refleja que en los archivos de resultados estadísticos de fin de año y durante el proceso de formación no se implementan acciones que potencialicen lo desempeños en los estudiantes, como lo establece el decreto 1290 del 2009 atendiendo a los propósitos formativos que debe tener la evaluación en el aula, que en concordancia con Castro, Martínez, \& Figueroa, (2009) es aprendizaje para todos los sujetos que intervienen en ella. Las concepciones de evaluación en el docente frente a su experiencia en el aula no da cuenta del uso de prácticas evaluativas fundamentadas en el modelo pedagógico que establece el PEI del establecimiento educativo (modelo socio-constructivista), pues se incentiva a la memorización y acumulación de información; siendo necesario un aprendizaje significativo en el cual serán necesarias condiciones de tipo didáctica que según Ausubel (citado por Pozo J. , 1997) deben tener una organización lógica, secuencial y psicológica para que el estudiante logre comprenderlos fácilmente, mostrando la importancia en que el maestro planifique, organice y coordine el aprendizaje, pues de esta manera en términos de Woolfolk, ( 2006) servirá de mediador y estimulador del mismo; logrando mayor motivación y disposición para aprender. En las observaciones de clases se revela predominio de metodología tradicionales en evaluación e indica que existe la necesidad en los docentes de hacer seguimiento al proceso de aprendizajes en el aula de clase alineando los desempeños esperados y actividades realizadas, al igual que el seguimiento a su proceso de enseñanza cumpliendo también una función pedagógica de la evaluación en el aula tanto para el docente como para el estudiante como lo afirma Álvarez, (2007) el sujeto aprende con ella y a través de ella. En la entrevista a docentes manifestaron conceptos diversos sobre evaluación, unos la conciben como un proceso y otros como medición, sin embargo es de notar que predomina una concepción tradicional enmarcada en la medición del conocimiento, en la cual se conciben las pruebas objetivas y exámenes como instrumentos que permiten evidenciar la mayor cantidad de conocimiento, de acuerdo con Prieto M. , (2008) sólo indica cuánto sabe el estudiante.

En el submomento de reflexión-acción al hacer el contraste entre las interpretaciones sobre evaluación con lo planteado en el decreto 1290 de 2009 y en el PEI se encontró que en la práctica de aula de docentes no se evidencia en su totalidad la forma de llevar a cabo este proceso de acuerdo a lo establecido en estos documentos, así mimos se refleja que no existe unificación de concepto y uso de la evaluación en el aula acorde con los propósitos legales que enmarca los procesos evaluativos en el aula, además entre lo expresado y observado se evidencian contradicción en varios docente entre lo que se cree y lo que se hace en el ámbito evaluativo, lo que clarifica que el proceso evaluativo llevado a cabo por docentes del Centro Educativo el Yeso se orienta bajo concepciones conductistas que se encuentra inmerso en el diseño de la enseñanza, la construcción de las tareas académicas, en la toma de decisiones y también en los procesos de evaluación, pues de acuerdo a Marrero, (2010) las representaciones mentales regulan e intervienen en el proceso de enseñanza y aprendizaje que se dan en la educación, lo cual amerita una 
reflexión, análisis y comprensión por parte de docentes y todos aquellos que intervienen en el proceso formativo.

Valoración - acción, en este ultimo submomento se da a conocer el informe final correspondiente a la primera fase en el cual el contraste realizado entre instrumentos aplicados (entrevista, revisión de documentos, descripción de clases y observación de clases) permitió revelar fortalezas como: el reconocimiento de docentes sobre sus falencias en el proceso de evaluación, interés por aprender más sobre el proceso de evaluación, algunos docentes en sus prácticas de aula emplean metodologías acorde con el modelo pedagógico del establecimiento educativo; y aspectos por mejorar como: concepciones que tienen sobre evaluación del aprendizaje con respecto al carácter formativo y función pedagógica, la incoherencia en docentes sobre lo que dicen y hacen en el aula en concordancia con los fundamentos legales, la articulación de los referentes de calidad en los métodos e instrumentos de evaluación.

\section{Informe fase dos: ejecución}

El alcance que la CRA obtuvo en el desarrollo de la segunda fase fue la revisión de conceptualizaciones alrededor de evaluación, del aprendizaje y decreto 1290 que rige actualmente el sistema educativo de evaluación colombiano. En el primer submomento de esta fase las acciones realizadas al interior de la CRA giraron alrededor de las confusiones y dudas de los docentes frente al tema de evaluación por ello se hizo pertinente iniciar la revisión teórica con la lectura analítica del decreto 1290 del 2009. En las afirmaciones e interrogantes que realizaron al respecto tales como: "La evaluación tiene el objetivo de mirar cuanto ha aprendido el estudiante y en cuantos temas tiene dificultades. Así podemos hacer los planes de acción y profundización" "ila institución posee un modelo evaluativo completo?", "¿Evaluaciones no son sólo las escritas?! Si son solo las escritas, también debemos revisarlas e incluir preguntas del ser y hacer" entre otras, reflejaban la escaza reflexión que hacen los docentes sobre sus prácticas evaluativas y el poco conocimiento en las teorías que las sustentan, por lo cual se hace necesario el ejercicio continuo en el docente de auto reflexión y análisis sobre su accionar, articulado y comparado a la luz de las teorías que fundamentan el proceso enseñanza aprendizaje establecido en la institución para poder potencializarlo y luego modificarlo o reformularlo, permitiéndole tomar decisiones acordes a las necesidades en contexto, pues, en palabras de Pérez \& Gimeno, (1988), observando y reflexionando sobre la práctica se puede descubrir el origen del conocimiento que condiciona la acción. Así mismo, los docentes deben reflexionar en torno a las teorías que fundamentan el proceso de enseñanza aprendizaje para hacer selección minuciosa de mecanismos de evaluación que permitan al estudiante evidenciar lo que sabe hacer con lo que ha aprendido, valorando las competencias en su integralidad, pues según Álvarez, (2007) toda practica evaluativa que no forme, que no eduque y de la cual los sujetos no aprendan debe eliminarse, pero también analizar que se está enseñando para que exista articulación entre lo que se enseña y lo que se evalúa. Por otra parte, los docentes en sus interrogantes sobre el desempeño de los estudiantes en el desarrollo de actividades como "ise requiere de juegos con dinamismo físico para reflejar el desarrollo de habilidades? denotan el uso en la práctica de aula de acciones que impliquen movimiento físico para evidenciar el papel activo del estudiante, por lo cual se hace necesario que el docente comprenda que la experiencia activa del estudiante en el aula desde una visión socio constructivista del aprendizaje implica manipulación no solo de objetos sino mental de ideas mediante actividades que vinculen el pensamiento con la acción como contrastar, analizar, critica, establecer relaciones, interpretar, entre otras, en términos de Woolfolk, (2006) toda actividad física implica una actividad intelectual. Por otra parte, las dudas manifestadas por los docentes dieron paso a la reflexión teórica dando lugar a claridades en el grupo con respecto al proceso evaluativo. Luego de repetidas sesiones de trabajo se observó apropiaciones al respecto; Expresiones como

"La adecuada articulación entre modelo pedagógico y prácticas evaluativas hacen que el proceso de enseñanza - aprendizaje sea verdaderamente integral y formativo. Puesto que en dicha articulación debemos revisar la pertinencia de estos con el contexto en el que desarrollamos las clases diarias" (D2). "Realmente estábamos haciendo énfasis en la función social de la evaluación, pero ahora comprendo que en el proceso se necesitan ambas" (D4) lo confirman. Lo que indica que mediante la reflexión en la acción inicia un proceso de decisiones que implica responsabilidad y autonomía de quien enseña para llegar a comprender cómo están aprendiendo los estudiantes; qué necesitan aprender; dónde se necesita fortalecer conceptos y procesos, enriqueciendo el proceso formativo. En palabras de Perrenoud,( 2007) la responsabilidad y autonomía de quien enseña no se entienden sin una gran capacidad de reflexionar en la acción y sobre la misma.

En el submomento de reflexión- acción se creyó pertinente revisar el sistema de evaluación institucional, en el cual se encontró incoherencias evidentes al no presentar una meta definida hacia donde se dirige el proceso formativo de los estudiante, la didáctica de la evaluación que apunta al como evaluar no se apoya en los referentes de calidad, lo que indica que no evalúan desempeños, pese a que el modelo pedagógico establecido en el PEI articula las competencias (socio-constructivismo); así mismo se evidencia que los padres de familia solo reciben información mas no participan en la toma de decisiones que enriquecen el proceso formativo de los estudiantes. Por lo cual se hace necesario que los docentes reflexionen con base en el decreto 1290 del 2009, el modelo pedagógico establecido en el centro educativo, los estándares básicos de competencia y los diferentes teóricos que hacen referencia a una evaluación formativa para la respectiva reestructuración al sistema de evaluación. 
En el último submomento de esta fase, una vez realizadas la revisión detallada del Sistema Institucional de Evaluación (SIE) permitió revelar las fortalezas tales como: Registro de informe académico unificado y la Información periódica a los padres de familia sobre el desempeño de los estudiantes; también aspectos por mejorar como: clarificar la meta a alcanzar con respecto al proceso enseñanza aprendizaje en los estudiantes con respecto a lo establecido en los referentes de calidad y el modelo pedagógico del establecimiento, unificar criterios de promoción y evaluación de los estudiantes que integren el saber, hacer, ser y convivir en función de su contexto, diseño del registro escolar unificado, entre otros.

\section{Informe tercera fase: valoración y sistematización}

El alcance que la CRA obtuvo en el desarrollo de la tercera fase fue el cambio generado en concepciones evaluativas de docentes teniendo en cuenta el carácter formativo de la evaluación, evidenciado en el desempeño de los estudiantes. Lo cual se alcanzó durante el desarrollo de los diferentes submomentos.

En el primer y segundo submomento de esta fase, se hizo un análisis de situación inicial hallada en el centro Educativo con las orientaciones encontradas en el proceso investigativo, el cual reveló que docentes del centro educativo el yeso han iniciado un cambio con respecto a la coherencia entre lo que se piensan y hacen en el proceso evaluativo en el aula, evidenciado en el conocimiento del modelo pedagógico el cual facilito la aplicación de los referentes de calidad en las diferentes practicas evaluativas, así mismo la concientización en los docentes de consolidar criterios de evaluación de manera colectiva para que el proceso de formación en el estudiante se caracterice por su integralidad y continuidad. Además, el reconocimiento por parte de los docentes sobre la naturaleza formativa que caracteriza la evaluación en el aula favoreciendo el desarrollo de competencias en el estudiante y al docente permitiéndole una mejor planificación y orientación de todo el proceso enseñanza aprendizaje. Con respecto del desempeño del estudiantes se evidencia mayor participación del estudiante debido al sentido, significatividad y funcionalidad dada a las diferentes actividades desarrolladas durante el proceso enseñanza-aprendizaje mediante la interacción con su contexto social y cultura que le permite según Vygotsky, (1995) desarrollar y demostrar capacidades, habilidades y destrezas de forma permanente. El interés generado en el uso de las diferentes formas de evaluar que anteriormente no utilizaban permitiéndole desarrollar ciertas habilidades de pensamiento denominadas por Gardner, (1999) inteligencias. Lo que indica que se le ha dado sentido al papel del estudiante, considerándolo en concordancia con Ferreyra \& Pedrazzi, (2007) protagonistas participativos, críticos, y reflexivos del proceso de socio-auto apropiación del conocimiento situado.

En el informe final de esta fase mediante conversatorio con docentes permitió la valoración a los cambios en las concepciones evaluativas de docentes, se destacan principales aprendizajes: Selección de instrumentos de evaluación acorde con la finalidad que se pretende alcanzar, mejoramiento de los procesos de evaluación, descubrir en cada docente la creatividad, fortalezas y debilidades para abordar las clases en todas las áreas. Principales acuerdo expuestos por los docentes: asumir el compromiso de seguir trabajando en comunidad de reflexión acción para retroalimentar conocimientos, no olvidando el proceso iniciado y mantener los cambios alcanzados. En cuanto al cambio con respecto a la concepción sobre evaluación del aprendizajes, los docentes manifestaron que se logro comprender que la evaluación es integral y además formativa con el propósito de mejorar, en ella no solo se debe hacer referencia a lo cuantitativo sino incluyendo lo cualitativo. Así mismo, en cuanto a los tipos de evaluación atendiendo al agente evaluador, el uso de la Coevaluacion en el aula y las diferentes formas de evaluar no solo con pruebas escritas sino incluyendo la observación, participación en temas de estudio, entre otras. A partir del uso de la reflexión en torno a teorías sobre evaluación del aprendizaje se ha transformado en las prácticas de aula en los siguientes aspectos: las actividades evaluativas han pasado de ser memorísticas a dinámicas, creativas, logrando el interés en los estudiantes, los estudiantes trabajan más motivados y desarrollan habilidades, entre otros. Lo anterior evidencia la valoración realizada por docentes sobre la experiencia de investigación en el Centro Educativo El Yeso catalogada al interior de la CRA como positiva, con la firme intención de dar continuidad al proceso de transformación iniciado al interior del aula pero también en cada uno de los participantes en este estudio.

\section{Conclusiones}

La evaluación como medio de aprendizaje contribuye a la autoformación de docentes y estudiantes al interior del proceso enseñanza-aprendizaje (Álvarez, 2007). Puesto que va dirigido a la reflexión y toma de decisiones que apunten a potencializar el proceso educativo $y$ en consecuencia a construir calidad en la educación. Para lo cual fue importante el grado de compromiso investigativo y flexibilidad frente a los cambios que fueron necesarios realizar y asumir durante el proceso para alcanzar la meta propuesta.

A continuación se presentan las conclusiones que revelan los alcances obtenidos en el desarrollo del pro0ceso investigativo acorde con los objetivos trazados:

- La investigación permitió identificar en los docentes una concepción de evaluación relacionada con la teoría de aprendizaje conductista donde se califica sólo la memorización de información y procedimientos, pues de acuerdo a Rodrígez (2008) estos principios conductistas en las aulas se evidencian en la forma de enseñar, aprender $y$ evaluar; lo cual generó el reconocimiento de la relación existente entre éste enfoque y el bajo desempeño académico alcanzado por estudiantes, 
al no propiciar espacios que incentiven el desarrollo de habilidades de pensamiento de orden superior.

- El proceso de investigación realizado ayudó a valorar la importancia de la revisión documental, bibliográfica e institucional como fuente de selección de criterios conceptuales que permitieron la valoración del proceso evaluativo implementado en el establecimiento educativo, lo cual dio lugar al reconocimiento de deficiencias en el desarrollo de una adecuada evaluación integral y formativa. Además, generó la comprensión de la dependencia de las practicas evaluativas en relación al pensamiento de quienes orientan el proceso; por lo tanto, para dejar de propiciar practicas evaluativas tradicionales debe ser transformado el pensamiento de los actores que intervienen en la enseñanza y el aprendizaje en términos de Fulla (citado por Mellado, 2011) "los cambios en educación dependen de lo que piensan y hacen los profesores, algo tan simple y a la vez tan complejo" (p.11).

- El proceso de observación de prácticas de aula forjó en los docentes una actitud reflexiva, en términos de Ferreyra \& Pedrazzi (2007) reflexionando sobre el obrar humano con ciencia y con conciencia (p.28). Al comparar en cada ejercicio las descripciones de lo observado con los criterios conceptuales tomados en la revisión teórica y en cada uno de ellos se realizaron retroalimentaciones que resaltaban las fortalezas, se definían deficiencias y sugerencias para mejorar el estado critico encontrado; pues, según Pérez \& Gimeno (1988), observando y reflexionando sobre la práctica se puede descubrir el origen del conocimiento que condiciona la acción (p.55). de esta manera simple se abordo la complejidad del proceso de transformaciones de concepciones, lo cual propició la incorporación de este tipo de actividades en las prácticas educativas institucionales.

\section{Referencias bibliográficas}

Ahumada, P. (2001). La evaluación una concepción de aprendizaje significativo. Valparaíso: Universidad católica de Valparaíso.

Álvarez, J. (2007). Evaluar para conocer, examinar para excluir. Madrid: Morata.

Antón, M. (2012). Docencia universitaria: concepciones y evaluacion de los aprendizajes. Estudio de caso. Burgos: Universidad de Burgos.

Aparicio, J., Hoyos, O., \& Niebles, R. (2004). De Velázques a Dalí: las concepciones implícitas de los profesores sobre el aprendizaje. Redalyc. org, (3), 144-168.

Ballester, M. (2008). Evaluación como ayuda al aprendizaje. Barcelona: GRAO.

Brown, S., \& Glasner, A. (2007). Evaluar en la universidad: problemas y nuevos enfoques. Madrid: NARCEA.

Casanova, M. (1998). evaluacion: concepto, tipología y objetivos. En M Casanova, Evaluacion educativa (págs. 67-89). México: SEPMuralla.

Castro, H., Martínez, E., \& Figueroa, Y. (2009). Fundamentaciones y orientaciones para la implementacion del Decreto 1290 del 16 de abril de 2009. Bogotá: Ministerio de Educación Nacional.

Coll, C. (1997). Los fundamentos del currículum. En C. Coll, Psicología y Currículum (pág. 35). México: Paidos.

Coll, C., \& Solé, I. (2007). Los profesores y la concepción constructivista. En C. Coll, E. Martín, T. Mauri, M. Miras, J. Onrubia, I. Solé, y otros, El constructivismo en el aula (págs. 7-22).
Barcelona: GRAO.

Córdoba, F. (2006). La evaluación de los estudiantes: una discusión abierta. Revista iberoaméricana, 2-9.

Delgado, B. (2010). Didactica aplicada a la evaluación en el área de lengua castellana y literatura en educacion secundaria. Córdoba: Universidad de Córdoba.

Di Franco, M. (2010). Representaciones docentes en la evaluacion escolar. Universidad Nacional de la Pampa, Argentina.

Escoriza, J. (1998). Conocimiento psicológico y conceptualización de las dificultades de aprendizaje. Barcelona: Universidad de Barcelona.

Elliot, J. (2000). El cambio educativo desde la Investigación Acción. Madrid: Morata.

Ferreyra, H., \& Pedrazzi, G. (2007). Teorías y enfoques psicoeducativos del aprendizaje. Argentina: Ediciones Novedades Educativas.

Gardner, H. (1999). Mentes extraordinarias: cuatro retratos para descubrir nuestra propia. Barcelona: Kairos.

Hernández, R. (2003). Evaluación del aprendizaje significativo en el aula cuadernos para la enseñanza del español II. Costa Rica: Universidad Estatal a Distancia .

Lafourcade, P. (1992). Investigación - acción. Kluwer Academic Press publisher, 1-8.

Marrero, J. (2010). El pensamiento reecontrado. Barcelona: Octaedro.

Mellado, V. (1996). Concepciones y prácticas de aula de profesores de ciencia, en formación inicial de primaria y secundaria. Enseñanza de la ciencia. Investigación y experiencias didácticas , 14 (3), 289302.

Mellado, V. (2011). Formación del profesorado de Ciencias y buenas prácticas: el lugar de la innovación y la investigación didáctica. En A. Caamaño, Física y Química. Investigación, innovación y buenas prácticas (Vol. III, págs. 11-28). Barcelona: GRAÓ.

Mora, A. (2004). La evaluación educativa: concepto, períodos y modelos. Revista Electrónica "Actualidades investigativas en educación", 4 (002), 2-24.

Moreno, M., \& Azcárate, C. (2003). Concepciones y creencias de los profesores universitarios de matemáticas acerca de la enseñanza de las ecuaciones diferenciales. Enseñanza de las ciencias , 21 (2), 265-280.

Murillo, F. (2011). Investigación acción. Madrid: Universidad Autónoma de Madrid.

O'shanahan, I. (1996). Enseñanza del lenguaje oral y las teorias implícitas del profesorado. San Cristobal de la laguna: Universidad de la Laguna.

Pérez, Á., \& Gimeno, J. (1988). Pensamiento y acción en el profesor: de los estudios sobre planificacion al pensamiento práctico. Dialnet $42,37-64$.

Perrenoud, P. (2007). Desarrollar la práctica reflexiva en el oficio de enseñar. Barcelona: GRAO.

Pozo, J. (1997). Teorías cognitivas del aprendizaje (Ouinta ed.). Madrid, España: Morata.

Prieto, M. (2008). Creencias de los profesores sobre evaluación y efectos incidentales. Revista de Pedagogía , 29 (84), 123-144.

Prieto, M. (2007). El constructivismo aplicado a la enseñanza de lenguas extranjeras. Sevilla: GRIN Verlag.

Rodrígez, M. (2008). Desarrollo de estrategias de aprendizaje en los alumnos de la carrera de Ingeniería en Mecanización Agropecuaria de la Universidad de Ciego de Ávila a partir de la disciplina física. Granada: Universidad de Granada.

Sanmartí, N. (2007). 10 ideas claves:evaluar para aprender. Barcelona: GRAO.

Valles, M. (1997). Técnicas cualitativas de investigación social. Madrid: síntesis.

Vázquez, F. (2012). La evaluación del aprendizaje en primaria y secundaria: los indicadores de evaluación. Espiral. Cuaderno del profesorado, $5(\mathbf{1 0}), 30-41$.

Vasilachis, I. (2006). Estrategias de Investigación Cualitativa Barcelona: Gedisa, S.A.

Vergara, C. (2011). Concepciones de evaluación del aprendizaje de docentes chilenos destacados de educación básica. Acción pedagógica, 06-18.

Vidales, I. (2005). Prácticas de Evaluación escolar en el nivel de educación primaria del Estado de Nuevo León. Estado de Nuevo León: Colegio de Estudios Científicos y Tecnológicos del Estado de Nuevo León.

Vygotsky, L. S. (1995). Pensamiento y Lenguaje. Teoria del desarrollo cultural de las funciones psiquicas (faustos ed.). (M. M. Rotger, Trad.) Buenos Aires: Psikolibros.

Woolfolk, A. (2006). Psicología Educativa. México: PEARSON. 